
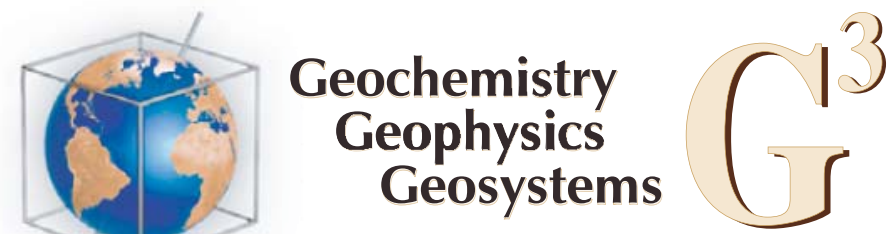

\title{
Polarized Earth's ambient microseismic noise
}

\section{Schimmel}

Institute of Earth Sciences Jaume Almera, CSIC, Lluis Sole i Sabaris s/n, E-08028 Barcelona, Spain (schimmel@ictja.csic.es)

\section{E. Stutzmann}

Institut de Physique du Globe de Paris, UMR-CNRS 7154, PRES Sorbonne Paris Cite, 1 rue Jussieu, F-75005 Paris, France (stutz@ipgp.fr)

\section{F. Ardhuin}

Laboratoire d'Océanographie Spatiale, Ifremer, BP 70, F-29280 Plouzané, France (ardhuin@shom.fr)

\section{J. Gallart}

Institute of Earth Sciences Jaume Almera, CSIC, Lluis Sole i Sabaris s/n, E-08028 Barcelona, Spain (jgallart@ictja.csic.es)

[1] We quantify, analyze, and characterize the frequency-dependent microseismic noise recorded by worldwide distributed seismic stations. Microseismic noise is generated through the interaction of ocean waves. It is the strongest ambient noise, and it is observed everywhere on Earth. We introduce a new approach which permits us to detect polarized signals in the time-frequency domain and which we use to characterize the microseismic noise. We analyze 7 years of continuous seismograms from the global GEOSCOPE network. Microseisms are dominated by Rayleigh waves, and we therefore focus on elliptically polarized signals. The polarized signals are detected in the time-frequency domain through a degree of polarization measure. We design polarization spectra and show that microseismic noise is more strongly polarized than noise in other frequency bands. This property is used to measure the directions of the polarized noise at individual stations as a function of time and frequency. Seasonal variations are found for the back azimuths and for the number of polarized signals at many stations. We show that the back azimuth directions are robust measurements that point toward the source areas computed from ocean wave models.

Components: 8100 words, 11 figures.

Keywords: microseismic noise; polarization; primary and secondary microseisms; seismology.

Index Terms: 7255 Seismology: Surface waves and free oscillations; 7299 Seismology: General or miscellaneous.

Received 15 April 2011; Revised 6 June 2011; Accepted 6 June 2011; Published 22 July 2011.

Schimmel, M., E. Stutzmann, F. Ardhuin, and J. Gallart (2011), Polarized Earth's ambient microseismic noise, Geochem. Geophys. Geosyst., 12, Q07014, doi:10.1029/2011GC003661.

\section{Introduction}

[2] Seismic background noise is omnipresent and recorded in a broad frequency band, from few milli Hertz to several tens of Hertz. The weakest and strongest known ambient noise are the hum and the microseisms (Figure 1), respectively. The hum [e.g., Suda et al., 1998; Tanimoto et al., 1998; Roult and Crawford, 2000; Rhie and Romanowicz, 2004] consists of continuously excited free oscillations of the Earth at about $5-20 \mathrm{mHz}$ while the microse- 
isms are dominantly Rayleigh waves observed at 0.04-1 Hz.

[3] Their precise source mechanisms are still under debate but related to atmospheric perturbations and ocean infragravity and gravity waves. Microseisms are split into primary (PM) and secondary microseisms (SM). The primary microseisms (PM in Figure 1) have the same frequencies as the ocean gravity waves and are generated through breaking near the shore and interaction of ocean waves with the sloping sea floor [Hasselmann, 1963]. The primary microseisms are strongest for frequencies between $0.05 \mathrm{~Hz}$ and $0.08 \mathrm{~Hz}$. The secondary microseisms (SM in Figure 1) are the dominant signals. They are strongest at $0.1-0.16 \mathrm{~Hz}$ and have the double frequency of ocean waves. SM are caused by double-frequency pressure oscillations through the interference of waves with nearly opposite wave numbers, i.e., with opposite directions and similar frequencies [Longuet-Higgins, 1950; Hasselmann, 1963; Tanimoto et al., 2006; Kedar et al., 2007; Ardhuin et al., 2011; E. Stutzmann et al., Observing and modelling seismic noise, submitted to Geophysical Journal International, 2011]. Among the broad wave spectrum, the nonlinear interaction of wave trains with almost opposite wave numbers produce double-frequency pressure waves that can propagate efficiently to large depth below the water wave base which is the depth to which the ocean wave can move water. This phenomenon results in the generation of SM through coupling with the seabed in the deep waters. The SM consists mainly of fundamental mode Rayleigh waves between $0.1 \mathrm{~Hz}$ and $0.16 \mathrm{~Hz}$, while above that frequency range higher modes tend to dominate as shown by Haubrich and McCamy [1969] from the analysis of wave number-frequency spectra. P waves and core phases have been observed in addition to Rayleigh waves with array data for noise frequencies of about $0.1-1.4 \mathrm{~Hz}$. The source areas of these body waves are mainly in the deep sea, as shown by seismic data analysis [Koper and de Foy, 2008; Gerstoft et al., 2008; Koper et al., 2009; Landes et al., 2010]. It is still under debate whether the observed surface waves are also generated in the deep ocean [Longuet-Higgins, 1950; Webb and Constable, 1986; Cessaro, 1994; Stehly et al., 2006; Chevrot et al., 2007; Kedar et al., 2007] or only in the near-coastal shallow waters [e.g., Friedrich et al., 1998; Bromirski and Duennebier, 2002; Schulte-Pelkum et al., 2004; Rhie and Romanowicz, 2006; Gerstoft and Tanimoto, 2007; Yang and Ritzwoller, 2008]. Comparisons of ocean wave spectra from offshore and nearshore buoys with SM at ocean bottom or inland seismic stations at the Oregon coast show that most of the microseisms are excited in nearshore areas close to California [Zopf et al., 1976; Bromirski and Duennebier, 2002].

[4] Friedrich et al. [1998] used two arrays, each array consisting of several seismic sensors deployed over a large area, and they observed stable sources of SM that are independent of the storms in the Norway Sea. The stable sources are explained by colliding ocean waves through interference with swell reflection at the coast. Several simultaneous sources can develop through this mechanism [Gerstoft and Tanimoto, 2007; Chevrot et al., 2007; Ardhuin et al., 2011]. Alternatively, the SM can also be excited by opposing wave interferences due to fast moving storms with group velocities faster than the ocean waves of about $5-10 \mathrm{~m} / \mathrm{s}$ or due to opposing swell from different storms [Longuet-Higgins, 1950; Kedar et al., 2007; Ardhuin et al., 2011].

[5] A seasonal variability of the SM has been reported, for instance by Aster et al. [2008] who further show that microseism power series correlate with continental-scale station distribution. Besides the seasonal variability there exist also a latitudinal dependence of seismic noise as observed from time-frequency spectra [Stutzmann et al., 2009]. They also show that continental stations can record microseisms generated several thousands of $\mathrm{km}$ away. Tanimoto et al. [2006] show, using data from Southern California, seasonal variations of the horizontal to vertical amplitude ratios $(\mathrm{H} / \mathrm{Z})$ for Rayleigh waves. They attribute these changes to seasonal variations of the source areas that vary the relative excitation of higher modes to the fundamental mode Rayleigh waves. Their back azimuths do not seem to change through the year which may imply that the sources move to different water depth.

[6] Most analyses, so far, are based on noise recordings at seismic station arrays of regional scale with focus on particular coastal regions. Through different signal processing strategies, the correlated or coherent portions of the microseisms are extracted from the array to determine the back azimuths which point toward the source areas. However, surface wave back azimuth data do not contain information on the distance to the source and can only be used through some type of triangulation to constrain the source areas.

[7] In this study we use globally distributed stations from the GEOSCOPE network to characterize the microseisms through their polarization. We analyze the polarization in the time-frequency domain following the strategy of Schimmel and Gallart [2004] 


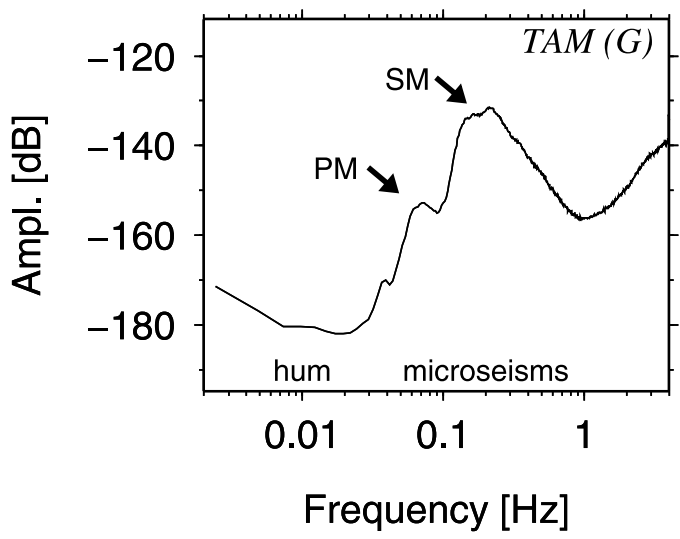

Figure 1. Vertical component power spectrum for 1 year of noise recorded at station Tamanrasset, Algeria (TAM). "Hum" and "microseism" mark the frequency ranges where they occur. The hum is not observed in Figure 1 . The primary and secondary microseismic peaks are marked by PM and SM, respectively. Other noise sources occur in all frequency bands.

on individual three component stations. Noise polarization studies are rare since it is not straightforward to extract the polarized signals from the multitude of signals. We show that we can measure a consistent seasonal variability of the number of detected polarized signals and their back azimuths from particle motion at each individual station. This is due to global seasonal variations of source areas as also shown by maps of theoretically expected SM generation areas which have been computed from global ocean wave models.

\section{Data and Method}

\subsection{Data}

[8] We use continuous waveform data from the global seismic network GEOSCOPE (Figure 2) to extract the frequency-dependent noise polarization from continuous three-component records. The data from the different components are converted to ground velocity by removing the instrument response. We use the long-period data (1 sample per second) for the years 2001 to 2007 to obtain a database of continuous three-component records from 27 stations over 7 years.

\subsection{Polarization Approach}

[9] The polarization describes the three-dimensional particle ground motion at the receiver considering seismic records along the three directions (northsouth, east-west, and vertical up). Directions to the incoming waves, called back azimuth (BAZ), and wave types can be inferred from the polarization. Microseisms are Rayleigh wave dominated [e.g., Haubrich et al., 1963; Tanimoto et al., 2006] which have elliptical polarization. In the ideal case, the ellipse stands in the vertical plane which connects the sensor and the source.

[10] For our polarization computation all individual records are transformed into the time-frequency domain. We use the S transform [Stockwell et al., 1996] for the time-frequency decomposition of each trace. The $\mathrm{S}$ transform is a multiresolution analysis which is related to the continuous wavelet transform [e.g., Ventosa et al., 2008], but which has Fourier properties similar to the Short Time Fourier Transform. We employ the $\mathrm{S}$ transform since the window size is scaled with period and since it uses Fourier frequencies and Fourier transform properties. For the analyses in this paper we use Gaussian-shaped windows with $2 \sigma=3 T$ where $\sigma$ and $T$ are the standard deviation and period, respectively. For a $10 \mathrm{~s}$ period signal this means that the $2 \sigma$ width of the Gaussian window is $30 \mathrm{~s}$. An alternative polarization

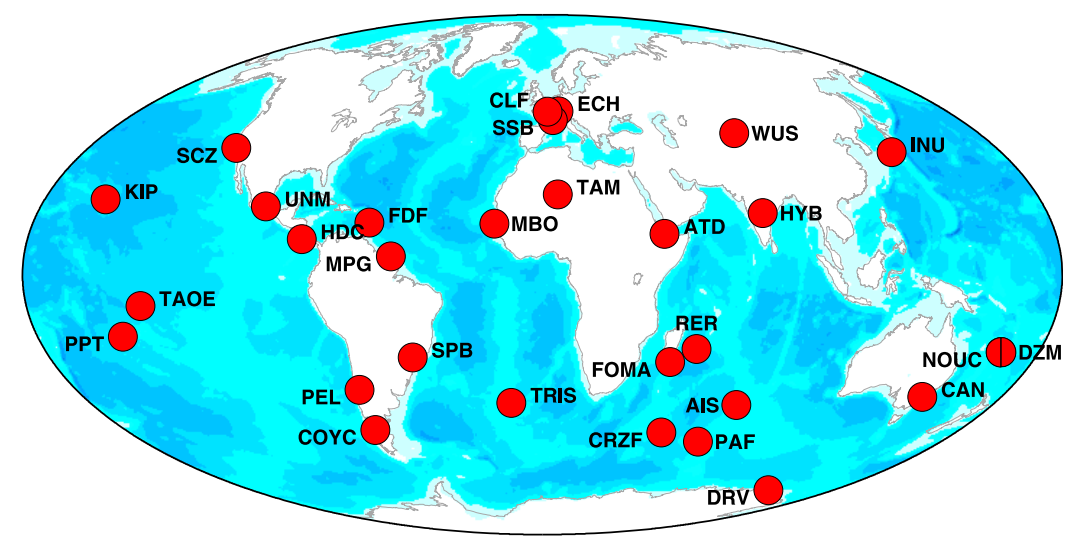

Figure 2. Global station map with the GEOSCOPE stations used in this publication. Station information and data can be downloaded from the GEOSCOPE Web page (http://www.geoscope.fr). 


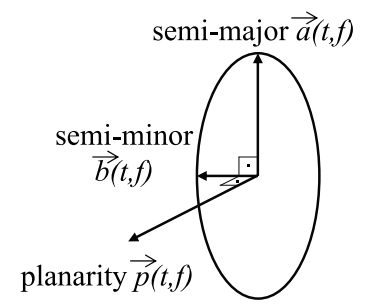

instantaneous ground

a)

motion ellipse

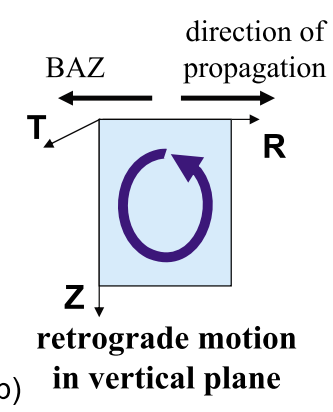

b)

Figure 3. (a) The semimajor vector $\vec{a}(t, f)$, semiminor vector $\vec{b}(t, f)$, and planarity vector $\vec{p}(t, f)$ are plotted together with the instantaneous polarization ellipse. All three vectors are mutually perpendicular. $\vec{a}(t, f)$ and $\vec{b}(t, f)$ are in the plane of the motion ellipse, while $\vec{p}(t, f)$ is perpendicular to the plane. (b) The expected particle motion for fundamental mode Rayleigh waves is elliptical with retrograde orientation in the vertical plane which connects the source and receiver. This is used to find the back azimuth (BAZ) to the source area without $180^{\circ}$ ambiguity.

method is the time domain covariance approach [e.g., Schulte-Pelkum et al., 2004] which is usually used with large windows to provide stable measures in the presence of one single stationary source. Shorter windows can also be used in the time domain as for instance through analytic signal theory [e.g., Schimmel and Gallart, 2003]. However, the timefrequency approach used here is more adaptive and better tackles nonstationary data and multiple sources due to the frequency-dependent analysis of short multiresolution windows.

[11] Polarization attributes, such as the semimajor and semiminor axis (Figure 3a) of the ellipse that best fits the ground motion, are determined through an eigen analysis of spectral matrices which have been constructed from the time-frequency representation of the three component seismograms. The polarization attributes are used to construct the degree of elliptical polarization.

[12] Our approach is to measure the instantaneous degree of polarization [Schimmel and Gallart, 2003, 2004, 2005] and BAZs of Rayleigh waves as function of time and frequency. The degree of polarization (DOP) is an instantaneous quality measure based on the stability of an arbitrary polarization state with time. It is based on the fact that a highquality signal should not vary its polarization through the course of the signal or equivalently through a small sliding data window. We measure the variability of polarization in terms of unit vector projections as shown in the expression for the DOP $c(t, f)$

$$
c(t, f)=\left(\frac{1}{N(f)} \sum_{\tau=t-\frac{T(f)}{2}}^{t+\frac{T(f)}{2}}\left|\frac{\vec{m}(t, f)}{|\vec{m}(t, f)|} \cdot \frac{\vec{p}(\tau, f)}{|\vec{p}(\tau, f)|}\right|^{\nu_{1}}\right)^{\nu_{2}}
$$

with mean vector $\vec{m}$

$$
\vec{m}(t, f)=\frac{1}{N(f)} \sum_{\tau=t-\frac{T(f)}{2}}^{t+\frac{T(f)}{2}} \frac{\vec{p}(\tau, f)}{\vec{p}(\tau, f) \mid}
$$

and planarity vector $\vec{p}(t, f) \cdot \vec{p}(t, f)$ is obtained by the vector cross product from the time-frequencydependent semimajor and semiminor vectors of the instantaneous ellipse which best describes the ground motion. The planarity vector (Figure $3 \mathrm{a}$ ) is perpendicular to the instantaneous plane of the ground motion ellipse. The unit planarity vector $\frac{\vec{p}(\tau, f)}{|\vec{p}(\tau, f)|}$ is expected to be constant for elliptical particle motion confined to a single plane. The planarity vector in equations (1) and (2) is replaced by the semimajor vector for linear or almost linear motion with linearity larger than 0.7 [Schimmel and Gallart, 2003, 2004].

[13] $T(f)$ defines the small sliding data window which we increase linearly with period in analogy to a multiresolution analysis. Here we use windows width $T(f)$ of four times the signal period $1 / f$. This means that ideally we detect signals with stable polarization during a minimum time of about 4 times the signal period. This value has been chosen intrinsically after some test runs as a compromise of considering the more significant larger duration signals and adaptability to nonstationary data with multiple signal sources. Using shorter windows will add more signals which are polarized during shorter durations. The total number of samples in each window is $N(f)$. The vector projections are measured with respect to their mean or median vector over the sliding data window. Vector $\vec{m}$ becomes largest for constant planarity vectors. Thus, $c(t, f)$ measures how well a signal is polarized.

[14] The exponents $\nu_{1}$ and $\nu_{2}$ are positive numbers to control the sensitivity through increasing the differences between polarized and less polarized signals. Usually, it is not important how these differences are increased and we simply use $\nu_{1}=\nu_{2}=\nu$. The exponents are important for waveform filtering purposes [Schimmel and Gallart, 2003, 2004, 2005] as they are used to tune the attenuation of less polarized signals. Here, the exponents can be used to control the number of polarized signals with a DOP above a 
preset threshold. Thus a high $\nu$ means that less signals are kept above a preset DOP threshold.

[15] We use unit vectors so that amplitudes do not bias $c(t, f)$, i.e., to consider equally large and small amplitude signals. Further, we adapt the degree of polarization to detect and to extract portions of noise which are elliptically polarized in a vertical plane. This is done by weighting $c(t, f)$ by the sine of the angle between the planarity vector and the vertical. The degree of polarization by Schimmel and Gallart [2004] has been modified to the degree of elliptical polarization with preferred vertical plane. $c(t, f)$ is a real number between 0 and 1 with 1 indicating a perfect polarized signal of elliptical particle motion in a vertical plane.

[16] The BAZs are determined for ground motions where the ellipse stands in a vertical plane (Figure 3b). There exists a $180^{\circ}$ ambiguity in the determination of the BAZ. We remove this ambiguity by assuming that the orientation of the ground motion ellipse is retrograde (Figure 3b) as is usually the case for fundamental mode Rayleigh waves recorded on rocky ground [Tanimoto and Rivera, 2005], and as is the case for GEOSCOPE stations [Stutzmann et al., 2000].

\section{Polarization Analysis}

[17] The data preprocessing and polarization analysis is performed in a semiautomated fashion. Problematic data have been identified and removed through different consistency and quality checks during the processing. Earthquake signals are also detected, but at the microseismic frequency range, these features last for shorter time spans than the microseisms from big storms. We therefore downweigh signals with BAZs which are clustered in time for a short duration rather than being distributed over several hours through a bootstrap resample analysis. Our final database consists of a huge matrix of time, frequency, degree of elliptical polarization and BAZ quadruples. These data are now used to analyze the microseismic noise.

\subsection{Polarization Spectra of Microseisms}

[18] First we count the occurrences of elliptically polarized signals as a function of frequency, station and year (2001-2007). Elliptically polarized signals are those which have a degree of elliptical polarization which is larger than a preset value. The exact value depends on the parameters used to determine the degree of polarization, but typical values range between 0.75 and 0.85 . We define polarization spectra as the distribution or number of polarized signals as a function of frequency. We determine the polarization spectra counting the number of detected elliptically polarized signals in $50 \%$ overlapping frequency bins of about $0.01 \mathrm{~Hz}$ width.

[19] Figure 4 shows the polarization spectra (black lines) for 9 stations. The absolute number of polarized signals are not presented since the purpose here is to show the relative distribution as a function of frequency. For comparison, we also show the power spectra of the vertical components (gray lines in Figure 4) at each station (for details see Stutzmann et al. [2009]).

[20] As shown in Figure 4, the PM and SM are clearly separated and visible as two distinct maxima in the polarization spectra. Thus, they are more often polarized than other noise at the surrounding frequencies. Furthermore, PM and SM are more clearly distinguished than in the power spectra where they cover a broader frequency range than in the polarization spectra. A direct comparison of the polarization spectra (black lines) and power spectra (gray lines) in Figure 4 shows that the PM and SM are more polarized at their respective lower frequencies. This is indicated for station UNM where the arrow point to maxima in the power spectra which occur at higher frequencies than the maxima of the polarization spectra. The ocean wave spectrum depends on the wind speed, wind duration, and fetch. For example, for a fully developed sea, Pierson and Moskowitz [1964] observed that the lower frequencies in the ocean wave spectra can only be excited by high wind velocities, such that the wind speed is at least $80 \%$ of the phase speed $g /(2 \pi f)$, combined with both a large fetch and a long duration. $g$ and $f$ are the acceleration due to gravity and the ocean wave frequency, respectively. The most polarized microseisms at the lower frequencies are probably generated by these ocean waves.

[21] The separation between PM and SM is visible for most stations and almost every day of the year. This is shown for station TAM (Tamanrasset, Algeria) in Figure 5 where one sees the time-frequencydependent polarization spectra. Figure 5 has been constructed after binning the measurements to polarization spectra per day using overlapping frequency bins which are $0.02 \mathrm{~Hz}$ wide. The red colors show when the largest amount of elliptical polarized signals occurs. The number of signals in each bin have been normalized to 1 and the color scale has 


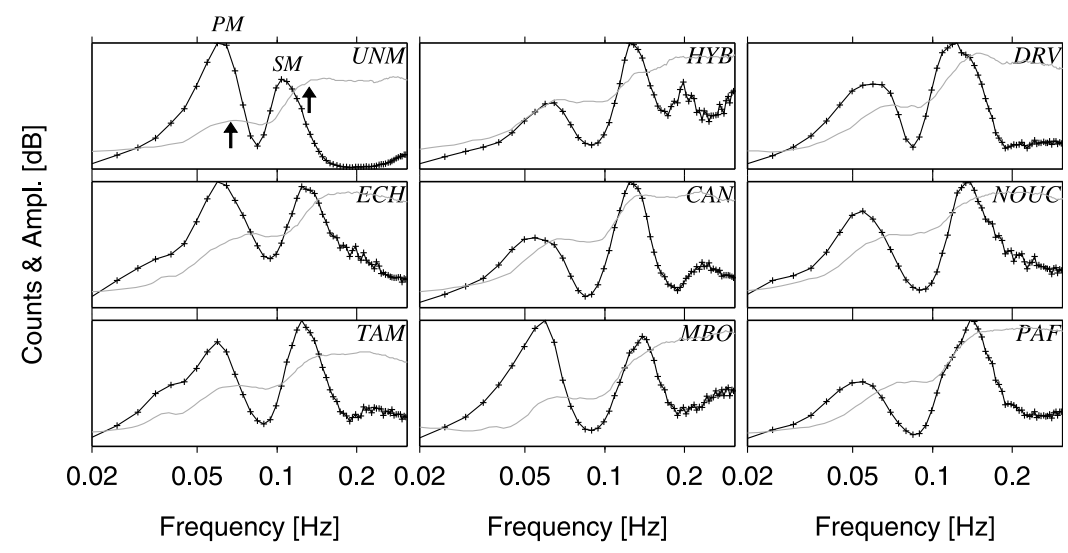

Figure 4. Polarization spectra (black) and power spectra (gray) for nine different stations: Unam, Mexico (UNM); Hyderabad, India (HYB); Dumont d'Urville, Antarctica (DRV); Echery, France (ECH); Canberra, Australia (CAN); Port Laguerre, New Caledonia (NOUC); Tamanrasset, Algeria (TAM); M'Bour, Senegal (MBO); and Port aux Francais, Kerguelen (PAF). PM and SM mark the primary and secondary microseisms in the polarization spectrum of UNM, while the arrows mark the corresponding maxima in the power spectra.

been saturated so that bins above 0.5 are shown in dark red to increase the visibility of smaller amplitude features. It can be seen from Figure 5 that there are clearly two red bands which correspond to the PM and SM which are detected through the entire year. Figure 5 shows a similar pattern for every year. At higher frequencies (about $0.25 \mathrm{~Hz}$ ) a third signal is observed. This signal is more pronounced during Northern Hemisphere summer and might correspond to a short-period double frequency peak [Bromirski et al., 2005]. We did not further analyze this last feature.
[22] Seasonal variations can be observed on the number of polarized signals as shown for the stations ECH (Echery, France), TAM (Tamanrasset, Algeria), CAN (Canberra, Australia) and PAF (Port aux Francais, Kerguelen Islands) in Figure 6. Plotted are the normalized moving averages obtained from $50 \%$ overlapping 10 day long windows and 7 years of data. The colored lines connect the averages per year. The blue and red colors are used to distinguish the PM $(0.05-0.09 \mathrm{~Hz})$ and SM $(0.1-0.14 \mathrm{~Hz})$ frequency bands used to count the polarized signals. The SM shows more seasonal variability than the

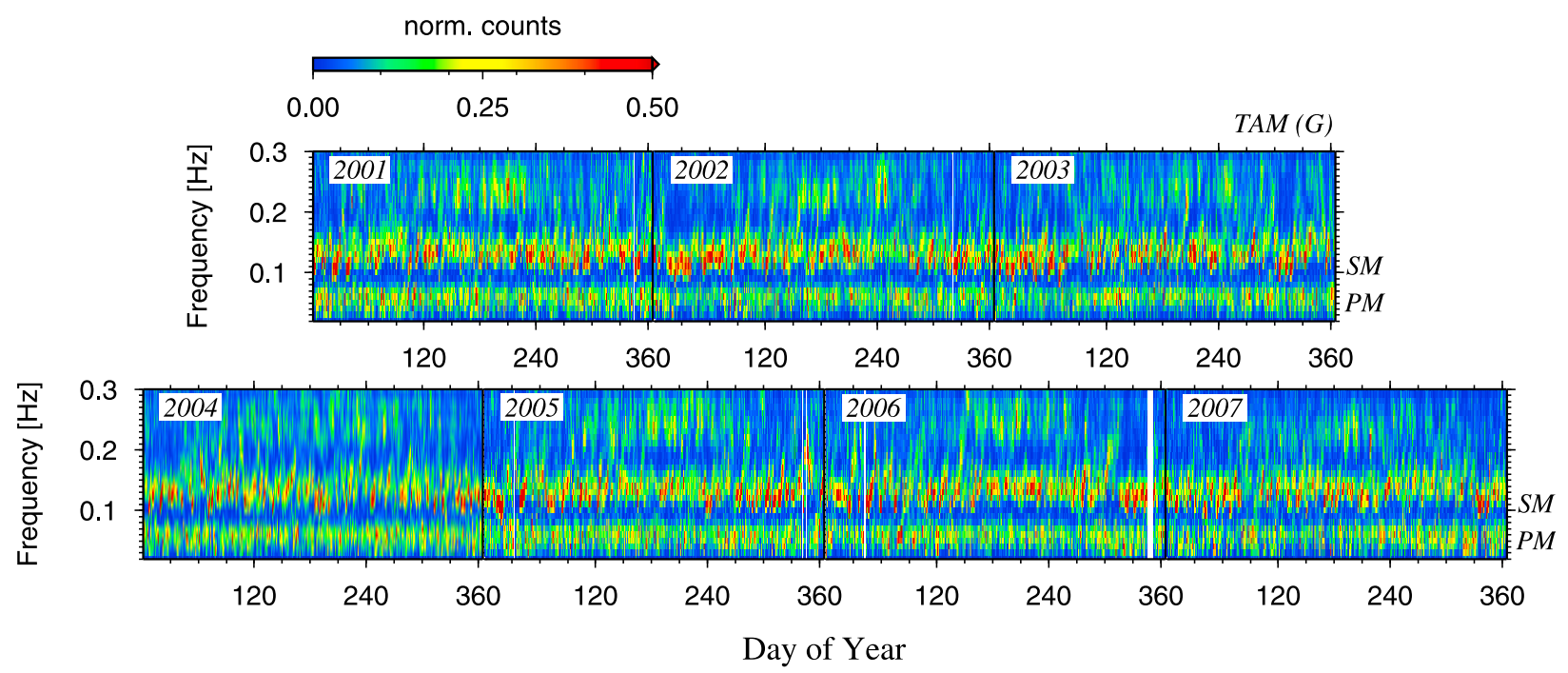

Figure 5. Polarization spectra as function of time and frequency for station TAM. The red colors correspond to the maximum number of polarized signals which are normalized to 1 . The color scale is saturated at dark red above 0.5 to increase the visibility of smaller amplitude features. The PM and SM are omnipresent and visible as horizontal red bands at about 0.07 and $0.14 \mathrm{~Hz}$, respectively. White is used whenever there is a day with no signal with degree of polarization above our threshold. 


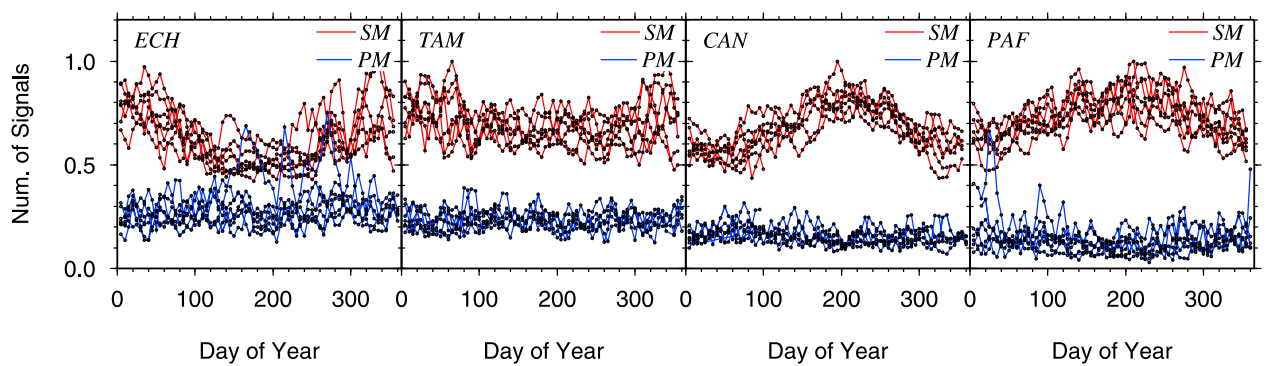

Figure 6. Number of elliptical polarized signals in a vertical plane as function of day of the year. Plotted are the normalized moving averages obtained from $50 \%$ overlapping 10 day data windows. The results for the 7 years are plotted on top of each other. Frequency bands are $0.05-0.09 \mathrm{~Hz}$ and $0.1-0.14 \mathrm{~Hz}$ for the primary (PM, blue lines) and secondary (SM, red lines) microseisms, respectively.

PM. One may attribute this observation to different microseismic source generation areas and mechanisms. The variability of the SM is correlated with the latitude of the station which decreases from left to right in Figure 6 . The first 2 stations are located in the Northern Hemisphere and the last 2 stations in the Southern Hemisphere. Most SM signals are observed for the local winter. TAM is located closer to the equator and shows therefore no pronounced variability, although there seems to be a slight tendency of stronger signals in the SM band during Northern Hemisphere winter. During the whole year there are more signals counted in the SM band than in the PM band which can be related to the fact that the SM are observed globally as the strongest microseisms and therefore expected to be better detected at the individual stations.

\subsection{Back Azimuths}

[23] The BAZ measurements are extracted from the elliptically polarized signals with particle motion in a vertical plane. The BAZs are obtained assuming that we observe fundamental mode surface waves with retrograde polarization (Figure $3 b$ ). This assumption permits to remove the $180^{\circ}$ ambiguity. Our polarization analysis is performed in the time-frequency domain and we therefore extract time-frequency-dependent BAZ measures.

[24] We first show 40 days of measurements for 3 stations from South and Central America to point to some common features in our data. Finally, we will analyze the 7 years of data.

\subsubsection{Back Azimuths and Ocean Wave Dispersion}

[25] Measured BAZs are plotted in Figure 7 (first, second, and third panels) as function of frequency and time for stations PEL (Chile), HDC (Costa
Rica) and UNM (Mexico). We plotted the most frequently measured BAZ of the most polarized signals within each hour. The most polarized signals are those which have a degree of elliptical polarization greater than 0.8 . White colors show the time-frequency locations without clearly polarized signals. The observed BAZ values are different by about $10^{\circ}$ to $50^{\circ}$ from the directions to the nearest coast. Similar events with BAZ values pointing to the Pacific ocean (not shown in Figure 7) are also observed at station MPG (French Guiana) which is located close to the Atlantic coast.

[26] In the frequency range of about $0.09-0.14 \mathrm{~Hz}$, we observe intermittent signals of linearly increasing frequencies with time and about constant BAZ. This pattern is similar to what is found in ocean wave records, and is due to the dispersive gravity wave velocities in deep waters [Darbyshire, 1952; Haubrich et al., 1963] with group velocity $u_{g}=$ $g T /(4 \pi)$, where $g$ is the acceleration due to gravity and $T$ the wave period. Ocean gravity waves at lower frequencies travel faster than at higher frequencies inducing travel time differences before microseismic waves are generated. Due to this dispersion, swell may meet at different places for different frequencies to couple into SM energy.

[27] The black lines in Figure 7 (first, second, and third panels) point to one of these linear dispersions which roughly begin at day 169 and $0.09 \mathrm{~Hz}$. These low-frequency signals can be explained by ocean waves which traveled about 4 days (day 165 is intercept time at $0 \mathrm{~Hz}$ ) in deep water before starting to generate microseisms on day 169 . The microseismic waves travel about 1000 times faster than the ocean waves and are much less dispersive. Therefore the storm excites ocean waves which travel from a few hours up to 15 days before microseisms are generated which then propagate within minutes to the recording stations. 


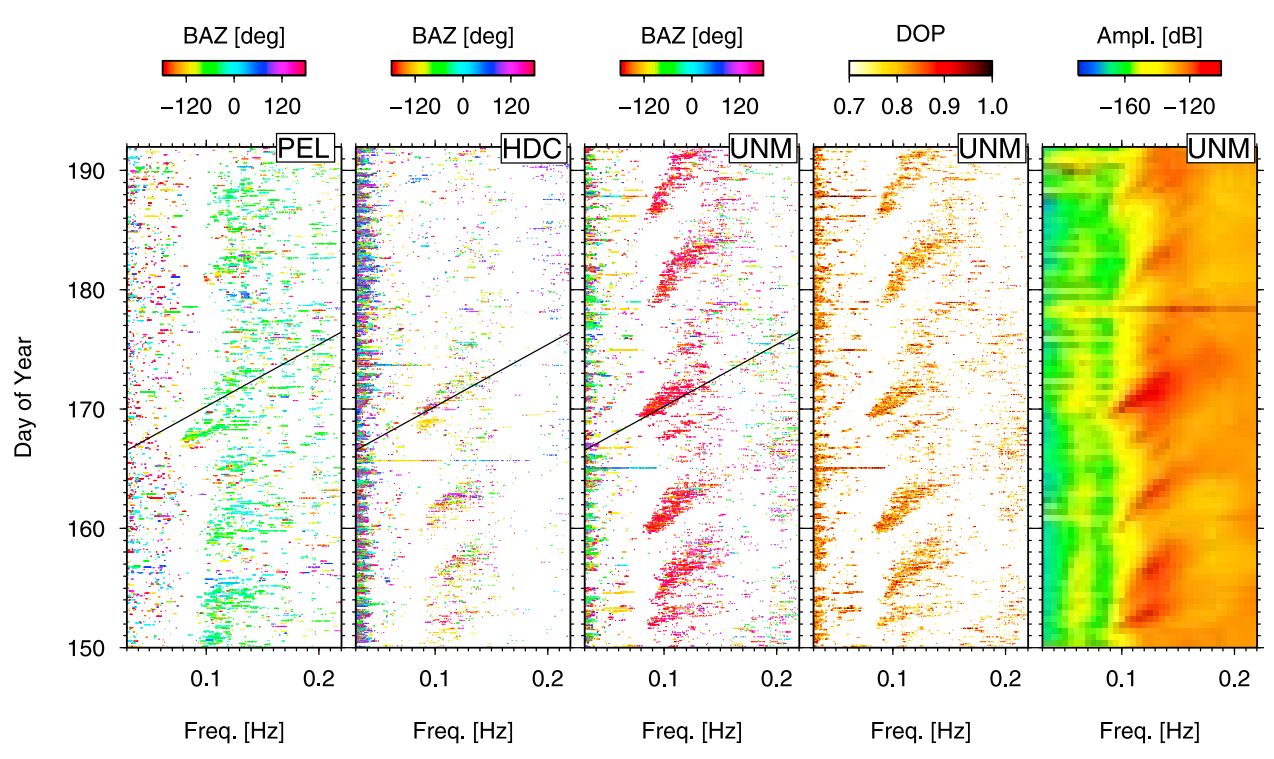

Figure 7. First, second, and third panels show examples of measured back azimuth (BAZ) during 42 days in June and July 2006 at three stations: Peldehue, Chile (PEL); Heredia, Costa Rica (HDC); and Unam, Mexico (UNM) as a function of frequency and time (day of the year in 2006). BAZs correspond to the most often measured direction per time-frequency bin. White area marks frequencies and days with few polarized signals. The fourth and fifth panels show the DOP and amplitude spectrum for station UNM.

[28] It seems that the seismic signals are recorded at later time at stations further to the north which is expected for the northward traveling swell which is the likely cause of these stripes as confirmed by satellite data and numerical models.

[29] The fourth and fifth panels of Figure 7 show the DOP and vertical component amplitude spectrum for data from station UNM. It is shown that the more polarized signals are indeed clustered along linear dispersion curves which are also seen in the amplitude spectra. The dispersions shown in Figure 7 are a common feature in our data and their occurrence on amplitude spectra is well known. It is the first time that these features are also observed on polarization data. Their simultaneous observation on spectra and polarization data further validates our approach since both methods are independent.

\subsubsection{Seasonal Variability of Back Azimuths}

[30] Figure 8 shows the number of polarized signals as function of BAZ and time throughout 7 years in the SM frequency band for station TAM. The polarized signals are counted in nonoverlapping bins of 1 day $\times 3^{\circ}$ size. The numbers of signals are normalized to 1 and the color scale has been saturated to increase the visibility of the less counted signals. It is seen that during Northern Hemisphere summer most of the waves arrive from the southern directions while during Northern
Hemisphere winter the waves come from the north. And again this pattern is repeated year by year and therefore related to seasonal climate. The polarized signals appear mainly in a band of about $90^{\circ}$ in BAZ. This width in BAZ is attributed to different sources of microseisms which happen over a broad area related to local winter and which all contribute to the recorded wavefield at the considered station. There is the same seasonality of BAZ observed at station TAM for the PM frequency band (not shown), however, slightly less well defined than for the SM.

[31] In order to investigate the frequency range, BAZs of the elliptically polarized noise are shown as a function of time and frequency in Figure 9 for station TAM. The BAZ is measured with respect to the north. Positive and negative angles are counterclockwise and clockwise, respectively. Figure 9 has been obtained after counting each day the occurrence of elliptically polarized signals in frequency-BAZ bins. The bins overlap by $50 \%$ and are $0.01 \mathrm{~Hz}$ and $3^{\circ}$ wide. Finally, we plot for each frequency the BAZ with the largest occurrence over the day. White is used whenever there has been no polarized signal. It can be seen from Figure 9 that the BAZ alternates between sources toward the northwest (green colors) during Northern Hemisphere winter and toward the south (red colors) during Northern Hemisphere summer. This variability is repeated every year and best visible at 


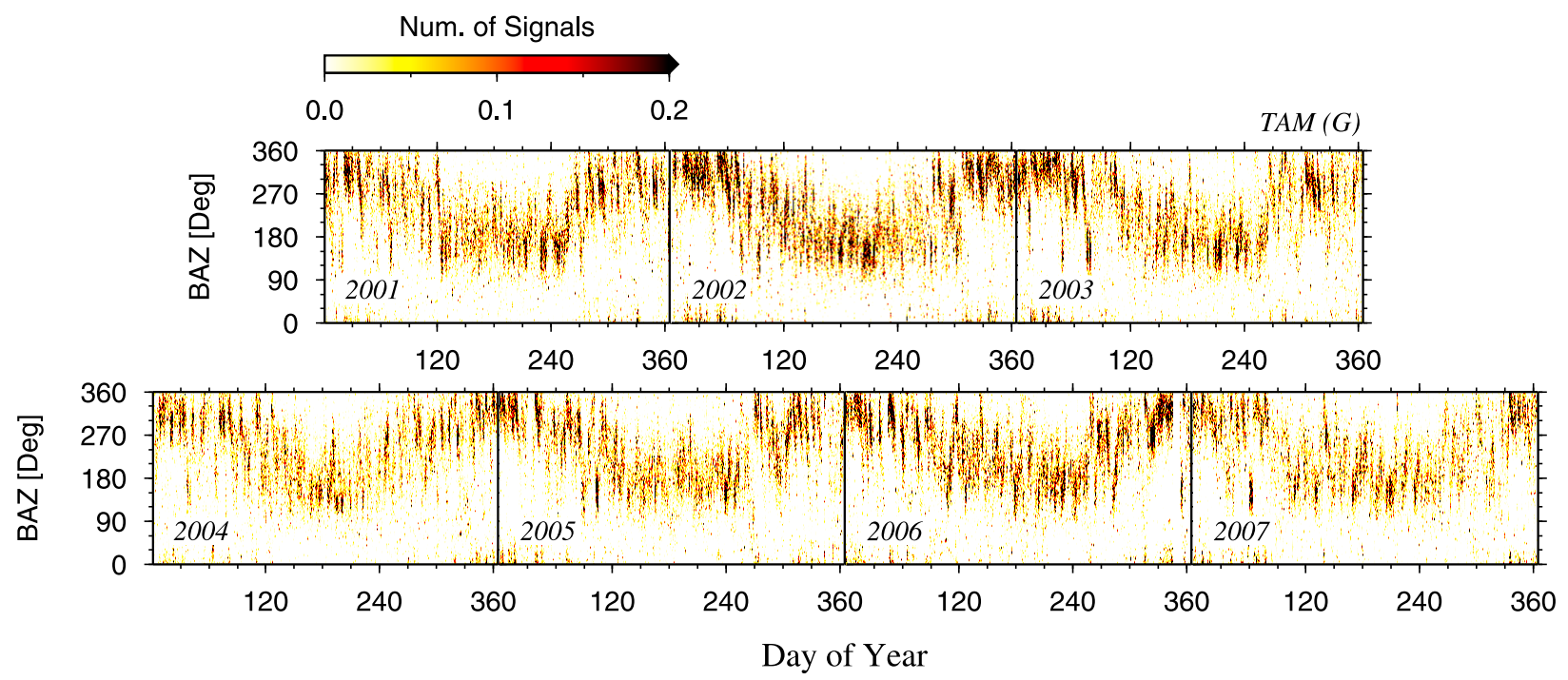

Figure 8. Number of elliptical polarized signals in a vertical plane as function of back azimuth (BAZ) and day of the year. The frequency band is $0.09-0.15 \mathrm{~Hz}$, and the nonoverlapping bins have a size of 1 day $\times 3^{\circ}$. The number of counted signals is normalized to 1 .

inland stations which are not obscured by any excitation at nearby shorelines. Figure 9 further shows that the frequencies for which less polarized signals are measured (white in Figure 9) are roughly aligned at the frequency $0.09 \mathrm{~Hz}$. This line seems to separate the PM and SM as seen from the polarization spectra.

[32] Figure 10 shows the BAZs as function of frequency and month for year 2006. The BAZs are shown by the angle and the frequencies by the radius. Inner and outer circle correspond to 0.05 and $0.25 \mathrm{~Hz}$, respectively. The colors show the number of signals at the corresponding directions and frequencies. It can now be seen how the BAZs change through the year. That is, from May to August the red spots point to the south while for November, December, January, and February one finds the red spots more to the north. Thus, although TAM shows no pronounced seasonality in number of detected signals (Figure 6) their BAZs clearly change over the year to point toward regions with local winter.

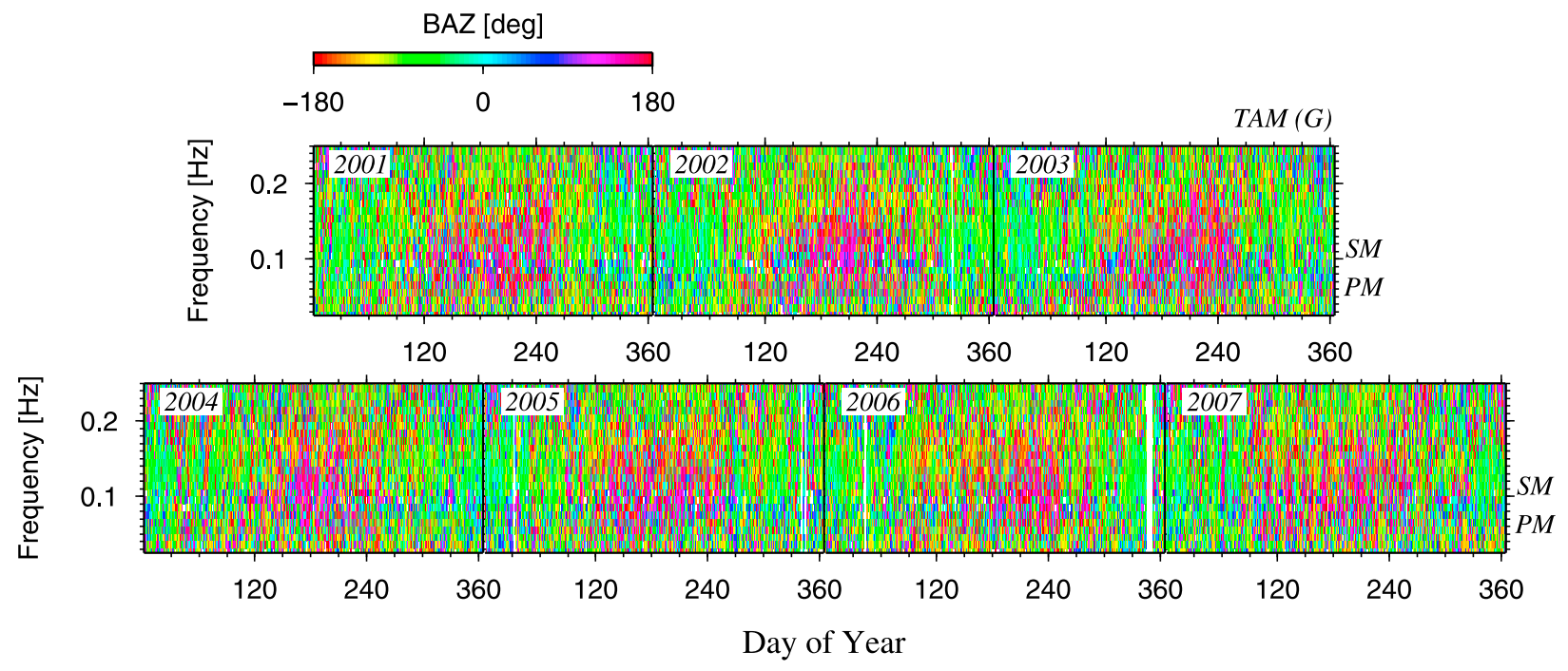

Figure 9. Most often measured back azimuth (BAZ) as function of time and frequency at station TAM. The seasonal dependence of BAZ is visible. BAZ points toward the south during Northern Hemisphere summer due to increased ocean storm activity in the Southern Hemisphere. 


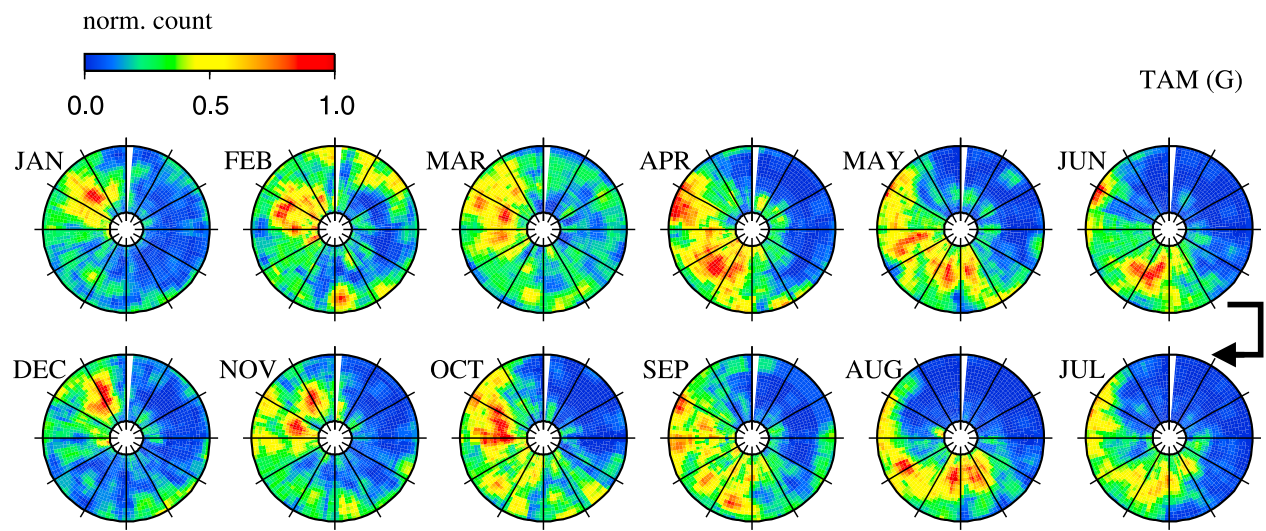

Figure 10. Variations of back azimuth (BAZ) per month in 2006 at station TAM. Red colors correspond to the maximum number of polarized signals, and the radius of each sphere corresponds to the frequency. The inner and outer circle are at 0.05 and $0.25 \mathrm{~Hz}$, respectively. The BAZ alternates between sources in the northwest during Northern Hemisphere winter and south during northern summer.

\subsubsection{Comparison With Source Generation Areas}

[33] To compare the measured BAZ directions with microseism source generation areas one needs to know the location of the seismic sources. For this reason we use here theoretically determined sources from an ocean wave model which is independent of our seismic data and which are also different from the significant wave height maps which generally are used to relate microseisms to ocean wave activity. As ocean model we use the Ifremer/ IOWAGA version of the WAVEWATCH III(R) code [Tolman, 2008], forced by wind and sea ice analyses from the European Center for MediumRange Weather Forecasts, and iceberg distribution analyzed by Ifremer. All the model settings and the expected model quality are fully documented by Ardhuin et al. [2010, 2011].

[34] The SM sources occur at the places where opposite traveling ocean wave trains meet with the same frequency. The superposition of these waves generates standing or partially standing waves which cause pressure fluctuations at doubled ocean wave frequency which efficiently couple into seismic energy. These sources are found through integrations of local ocean wave spectra over azimuths for all the oceans. The employed theory further accounts for resonance terms [LonguetHiggins, 1950; Kedar et al., 2007] which depend on water depths to modulate the energy of the pressure fluctuations and therefore the resulting seismic sources.

[35] The source areas are determined from the modeling of microseismic generation by random ocean waves, including ocean wave reflections from shorelines (continents and islands) and icebergs by Ardhuin et al. [2011]. For details of the numerical modeling and theory see Ardhuin et al. [2011] and Stutzmann et al. (submitted manuscript, 2011) who use the theory based on Longuet-Higgins [1950] and Hasselmann [1963] for SM generation.

[36] The disks in Figure 11 are SM source maps which are centered at stations CAN, TAM and SSB. Mapped are the seismic sources for frequencies $0.09-0.15 \mathrm{~Hz}$ using an azimuthal equidistant projection and a maximum distance of $60^{\circ}$. The outer rings in Figure 11 contain the normalized number of measured elliptical polarized signals in a vertical plane for the same frequencies as a function of BAZ. Both, the number of polarized signals and sources are averaged over 1 month for January, April, July and October to represent the four seasons.

[37] It is seen that the polarization directions mostly coincide with a source in that direction. Further, the source generation areas show the same seasonal variations as the BAZs. A complete match between polarization data and ocean wave models is not expected due to the limitations of each approach. The detection of polarized signals from seismic wavefield fluctuations due to waves which simultaneously arrive from the different sources is difficult and likely lead to null observations. The polarization approach is therefore expected to be biased in the sense that waves from few isolated sources should be detected better than for many simultaneous sources. Further, the DOP is an amplitude unbiased approach and the number of polarized signals depends on the duration rather than the energy of the source. The energy is only 


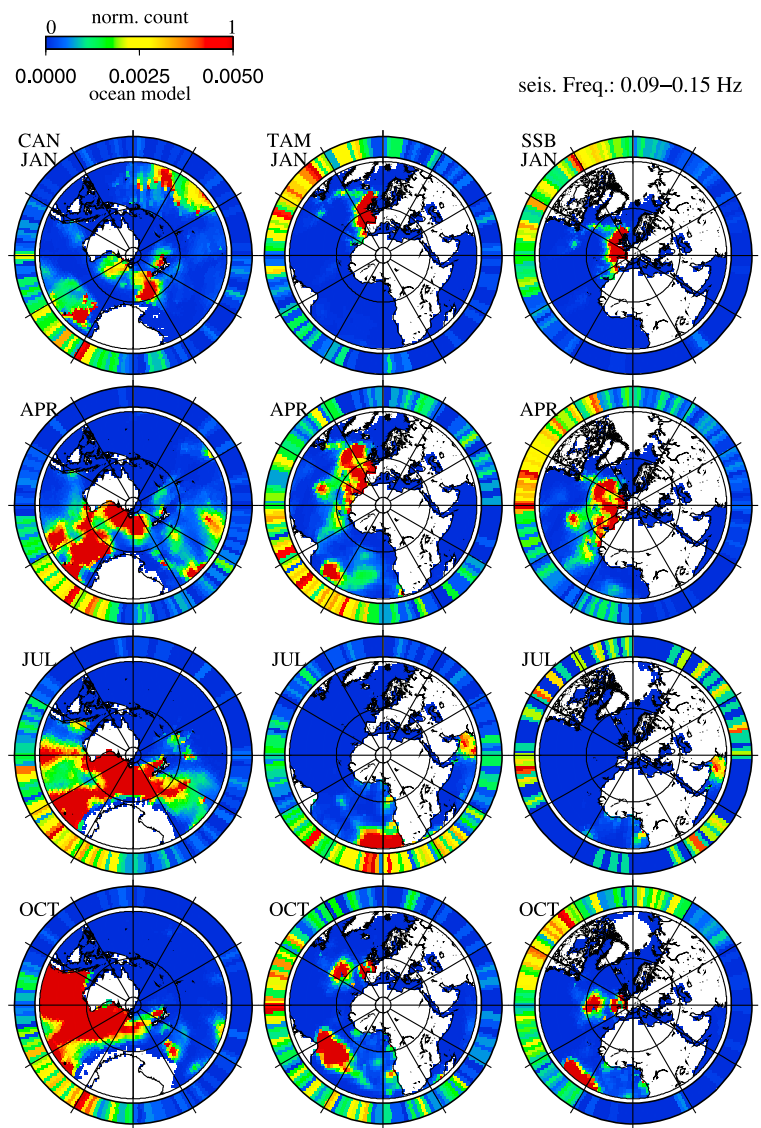

Figure 11. Normalized number of polarized signals as function of BAZ (outer rings) and map with theoretically determined SM generation areas. Maps are centered at stations CAN, TAM, and SSB, and the azimuthal equidistant projection permits a direct comparison with the measured BAZ. Sources and BAZ values are averaged for 1 month using January, April, July, and October of 2007 to represent the four seasons.

important in the sense that the weaker the amplitudes of the waves the more sensitive they are to other noise. These reasons may explain why the sources to the NE of CAN in January or to the NW of TAM in April have no or few matching BAZ values.

[38] Concerning the ocean wave models, they have been validated with success by Ardhuin et al. [2011] and Stutzmann et al. (submitted manuscript, 2011) by modeling seismic noise spectral variations over the year.

\section{Discussion}

[39] The PM and SM are characterized by their large amount of elliptically polarized signals. The comparison of power and polarization spectra show that these signals are more polarized at their respective lower frequencies than at their higher frequencies (Figure 4). For a fully developed sea lower frequencies are primarily excited by high wind velocities and large fetch [Pierson and Moskowitz, 1964]. Thus the more elliptically polarized signals are probably due to large storms which blew for a long time over a large area. These long-period ocean waves may travel long distances before generating microseisms. At their respective higher frequencies, the microseisms are probably less polarized due to more variable storms of shorter duration and weaker strength. Further, the ocean wave spectrum decays with highest frequencies lost first when moving away from a source generation area $[\mathrm{Webb}$, 1992]. Therefore, higher-frequency components of microseisms are expected to be weaker and the multitude of small-amplitude Rayleigh waves would interfere almost at any time and place which destroys their stable polarization at the stations. This may explain the differences between the polarization and power spectra (Figure 4).

[40] Striking is also our observation of an increased number of polarized signals in the SM frequency band for local winter (Figure 6) while the number of polarized signals seems to stay constant for the PM. This behavior is attributed to the different source generation mechanism. If we assume that an increased swell activity is responsible for this observation then this same swell seems not to contribute significantly to the PM. This can be explained if the SM are generated in another source area than the PM. Otherwise, we would expect a similar increase of PM activity.

[41] The linear dispersion of ocean waves, as shown in Figure 7 is a common feature in our polarization data set. The dispersion is well known and has been observed in the time-frequency amplitude spectra of several studies [e.g., Friedrich et al., 1998; Bromirski and Duennebier, 2002; Barruol et al., 2006; Gerstoft and Tanimoto, 2007; Chevrot et al., 2007; Aster et al., 2008]. So far, this is the first time that these features are also observed on polarization data from individual stations. Same type of striations are also seen in the time-frequency amplitude spectra of our data. The observation in polarization data, however, adds the BAZ as new information to this striations without the need of array analyses. The striations from Figure 7 are attributed to swell generated by Southern Hemisphere winter storms which travel northward along the South American coast. The slope of the striations is due to ocean wave dispersion and gives access to the distance between the microseismic source and the ocean wave storm area that can be 
several thousands of $\mathrm{km}$ away. The BAZ at each station points toward the SM source. A storm can generate microseisms at different locations and times, for example when SM are generated by the interference between incident and coastal reflected swell.

[42] The seasonal variation of source areas is observed year after year in the measured BAZs of individual stations. This dependence seems to be clearest at inland stations. The observed broad range of BAZ values of Figure 8 is due to multiple sources. The BAZs obtained by Schulte-Pelkum et al. [2004], Tanimoto et al. [2006], Gerstoft and Tanimoto [2007], and Yang and Ritzwoller [2008], among others, for stations in the western United States are stable throughout the year and point to the SW or SSW Pacific for the SM. We observe a similar constant BAZ at station SCZ (not shown). However, the number of polarized signals is higher in local winter than in summer. A seasonal variability is also seen by Tanimoto [2007] in the H/Z ratio which can be due to source regions which move between areas of different water depths to excite more or less higher-mode Rayleigh waves with respect to the fundamental modes.

[43] At the higher frequencies of the $\operatorname{SM}(0.3-1.4 \mathrm{~Hz})$ Koper and de Foy [2008] detect seasonal P wave and Rayleigh wave amplitude variations in Thailand which correlate well with the seasonal ocean wave height variations in the neighboring ocean. A clear seasonal variability of source regions at the respective winter has also been found for the PM by Stehly et al. [2006].

[44] The modeled SM source maps have been used since they are based on completely independent ocean wave data acquired mainly from satellites and buoys. The SM source maps are different from significant wave height maps as they take into account the physical process that generate SM [LonguetHiggins, 1950; Hasselmann, 1963; Kedar et al., 2007; Ardhuin et al., 2011; Stutzmann et al., submitted manuscript, 2011]. We compare the BAZ with SM source areas obtained from the global ocean wave model which include coastal reflections [Ardhuin et al., 2011]. We show a good agreement between seasonal variations of $\mathrm{BAZ}$ and seasonal variations of source areas confirming that the BAZ is a robust measure of the source direction. However, not all source areas are detected by the BAZs which we attribute to the fact that there are multiple sources spread over wide or different areas [e.g., Gerstoft and Tanimoto, 2007]. The simultaneous wave arrivals from different sources at the recording stations may destroy the polarization and we therefore measure the strongest sources.

\section{Conclusion}

[45] We have shown that our polarization approach is useful for analyzing seismic ambient noise. Attributes such as the time-frequency-dependent degree of polarization and BAZs have been used to characterize microseisms and to show their variabilities. We further show how to construct polarization spectra as function of frequency for stationary signals and as function of time-frequency for nonstationary signals. Altogether, these are important tools to localize, to characterize and to monitor climate induced microseisms through independent attributes obtained by individual three component stations. Monitoring interdecadal climate change should be possible using long-running seismic stations and the polarization can provide independent measures in studies which model wave climate.

[46] We analyzed 7 years of continuous data from the global GEOSCOPE seismic network. The PM and SM are clearly visible from the polarization spectra. The time-frequency-dependent arrival of microseisms due to the linear dispersion of ocean waves before they couple into seismic energy is a common feature in our data. Many stations show seasonal variations of the BAZs which show the same periodicity as the ocean wave models. Swells may travel potentially long distances before they couple into SM. Nevertheless, the microseisms source areas are related to the seasonal periodicity of swell which drives a seasonal periodicity of microseisms.

[47] We have shown that the noise polarization measured as a function of frequency on individual stations is an important tool to localize, to characterize and to monitor climate induced microseisms through independent attributes. This capability can be applied to design the deployment of temporal seismic ambient noise experiments, to correct bias due to the spatial random source assumption in ambient noise tomography, to identify optimal ocean bottom monitoring sites, and to reconstruct wave climate for which only few measurements at buoys are available. Finally, locating microseism sources may also pave new ways for imaging shallow Earth structures.

\section{Acknowledgments}

[48] This research has been possible due to support by GEOSCOPE, IPG-Paris, the consolider-Ingenio 2010 program 
CSD2006-00041 (Topo-Iberia), PII200830I202, ILink2010-0112, and CGL2009-09727 (Rifsis). M.S. acknowledges the GEOSCOPE and IPGP team for their invitations to several research stays. We are grateful to the Editor T. Becker, B. Romanowicz, and an anonymous reviewer for their constructive reviews which improved our manuscript. This is IPGP contribution 3184 .

\section{References}

Ardhuin, F., et al. (2010), Semi-empirical dissipation source functions for ocean waves. Part I: Definition, calibration, and validation, J. Phys. Oceanogr., 40, 1917-1941, doi:10.1175/2010JPO4324.1.

Ardhuin, F., E. Stutzmann, M. Schimmel, and A. Mangeney (2011), Ocean wave sources of seismic noise, J. Geophys. Res., doi:10.1029/2011JC006952, in press.

Aster, R. C., D. E. McNamara, and P. D. Bromirski (2008), Multidecadal climate-induced variability in microseisms, Seismol. Res. Lett., 79, 194-202, doi:10.1785/gssrl.79.2.194.

Barruol, G., D. Reymond, F. R. Fontaine, O. Hyvernaud, V. Maurer, and K. Maamaatuaiahutapu (2006), Characterizing swells in the southern Pacific from seismic and infrasonic noise analyses, Geophys. J. Int., 164, 516-542, doi:10.1111/ j.1365-246X.2006.02871.x.

Bromirski, P. D., and F. K. Duennebier (2002), The nearcoastal microseism spectrum: Spatial and temporal wave climate relationships, J. Geophys. Res., 107(B8), 2166, doi:10.1029/2001JB000265.

Bromirski, P. D., F. K. Duennebier, and R. A. Stephen (2005), Mid-ocean microseisms, Geochem. Geophys. Geosyst., 6, Q04009, doi:10.1029/2004GC000768.

Cessaro, R. K. (1994), Sources of primary and secondary microseisms, Bull. Seismol. Soc. Am., 84, 142-148.

Chevrot, S., et al. (2007), Source locations on secondary microseisms in western Europe: Evidence for both coastal and pelagic sources, J. Geophys. Res., 112, B11301, doi:10.1029/2007JB005059.

Darbyshire, J. (1952), The generation of waves by wind, Proc. R. Soc. London, Ser. A, 215, 299-328.

Friedrich, A., F. Krüger, and K. Klinge (1998), Oceangenerated microseismic noise located with the Graffenberg array, J. Seismol., 2, 47-64.

Gerstoft, P., and T. Tanimoto (2007), A year of microseisms in southern California, Geophys. Res. Lett., 34, L20304, doi:10.1029/2007GL031091.

Gerstoft, P., P. M. Shearer, N. Harmon, and J. Zhang (2008), Global P, PP, and PKP wave microseisms observed from distant storms, Geophys. Res. Lett., 35, L23306, doi:10.1029/2008GL036111.

Hasselmann, K. (1963), A statistical analysis of the generation of microseisms, Rev. Geophys., 1, 177-209.

Haubrich, R. A., and K. McCamy (1969), Microseisms: Coastal and pelagic sources, Rev. Geophys., 7, 539-571.

Haubrich, R. A., W. H. Munk, and F. E. Snodgrass (1963), Comparative spectra of microseisms and swell, Bull. Seismol. Soc. Am., 53(1), 27-37.

Kedar, S., et al. (2007), The origin of deep ocean microseisms in the North Atlantic Ocean, Proc. R. Soc. A, 464, 777-793, doi:10.1098/rspa.2007.0277.

Koper, K. D., and B. de Foy (2008), Seasonal anisotropy in short-period seismic noise recorded in South Asia, Bull. Seismol. Soc. Am., 98, 3033-3045, doi:10.1785/0120080082.
Koper, K. D., B. de Foy, and H. Benz (2009), Composition and variation of noise recorded at the Yellowknife seismic array, 1991-2007, J. Geophys. Res., 114, B10310, doi:10.1029/ 2009JB006307.

Landes, M., F. Hubans, N. M. Shapiro, A. Paul, and M. Campillo (2010), Origin of deep microseisms by using teleseismic body waves, J. Geophys. Res., 115, B05302, doi:10.1029/2009JB006918.

Longuet-Higgins, M. S. (1950), A theory of the origin of microseisms, Philos. Trans. R. Soc. London, Ser. A, 243, 1-35.

Pierson, W. J., and L. Moskowitz (1964), A proposed spectral form for fully developed wind seas based on the similarity theory of S. A. Kitaigordskii, J. Geophys. Res., 69, 5181-5190.

Rhie, J., and B. Romanowicz (2004), Excitation of Earth's continuous free oscillations by atmosphere-ocean-seafloor coupling, Nature, 431, 552-556.

Rhie, J., and B. Romanowicz (2006), A study of the relation between ocean storms and the Earth's hum, Geochem. Geophys. Geosyst., 7, Q10004, doi:10.1029/2006GC001274.

Roult, G., and W. Crawford (2000), Analysis of 'background' free oscillations and how to improve resolution by subtracting the atmospheric pressure signal, Phys. Earth Planet. Inter., 121, 325-338.

Schimmel, M., and J. Gallart (2003), The use of instantaneous polarization attributes for seismic signal detection and image enhancement, Geophys. J. Int., 155, 653-668, doi:10.1046/ j.1365-246X.2003.02077.x.

Schimmel, M., and J. Gallart (2004), Degree of polarization filter for frequency-dependent signal enhancement through noise suppression, Bull. Seismol. Soc. Am., 94, 1016-1035, doi:10.1785/0120030178.

Schimmel, M., and J. Gallart (2005), The inverse S transform in filters with time-frequency localization, IEEE Trans. Signal Processing, 53(11), 4417-4422, doi:10.1109/ TSP.2005.857065.

Schulte-Pelkum, V., P. S. Earle, and F. L. Vernon (2004), Strong directivity of ocean-generated seismic noise, Geochem. Geophys. Geosyst., 5, Q03004, doi:10.1029/ 2003GC000520.

Stehly, L., M. Campillo, and N. M. Shapiro (2006), A study of the noise from its long-range correlation properties, J. Geophys. Res., 111, B10306, doi:10.1029/2005JB004237.

Stockwell, R. G., L. Mansinha, and R. P. Lowe (1996), Localization of the complex spectrum: The $\mathrm{S}$ transform, IEEE Trans. Signal Processing, 44(4), 998-1001.

Stutzmann, E., G. Roult, and L. Astiz (2000), Geoscope station noise level, Bull. Seismol. Soc. Am., 90, 690-701.

Stutzmann, E., M. Schimmel, G. Patau, and A. Maggi (2009), Global climate imprint on seismic noise, Geochem. Geophys. Geosyst., 10, Q11004, doi:10.1029/2009GC002619.

Suda, N., K. Nawa, and Y. Fukao (1998), Earth's background free oscillations, Science, 279, 2089-2091.

Tanimoto, T. (2007), Excitation of microseisms, Geophys. Res. Lett., 34, L05308, doi:10.1029/2006GL029046.

Tanimoto, T., and L. Rivera (2005), Prograde Rayleigh wave particle motion, Geophys. J. Int., 162, 39-405.

Tanimoto, T., J. Um, K. Nishida, and N. Kobayashi (1998), Earth's continuous oscillations observed on seismically quiet days, Geophys. Res. Lett., 25, 1553-1556, doi:10.1029/ 98GL01223.

Tanimoto, T., S. Ishimaru, and C. Alvizuri (2006), Seasonality in particle motion of microseisms, Geophys. J. Int., 166, 253-266.

Tolman, H. L. (2008), A mosaic approach to wind wave modeling, Ocean Modell., 25, 35-47, doi:10.1016/j.ocemod.2008.06.005. 
Ventosa, S., C. Simon, M. Schimmel, J. J. Dañobeitia, and A. Manuel (2008), S-transform from a wavelets point of view, IEEE Trans. Signal Processing, 56, 2771-2780.

Webb, S. C. (1992), The equilibrium oceanic microseismic spectrum, J. Acoust. Soc. Am., 92, 2141-2158.

Webb, S. C., and S. C. Constable (1986), Microseism propagation between two sites on the deep seafloor, Bull. Seismol. Soc. Am., 76, 1433-1445.
Yang, Y., and M. H. Ritzwoller (2008), The characteristics of ambient seismic noise as a source for surface wave tomography, Geochem. Geophys. Geosyst., 9, Q02008, doi:10.1029/ $2007 \mathrm{GC} 001814$.

Zopf, D. O., H. C. Creech, and W. H. Quinn (1976), The wavemeter: A land-based system for measuring nearshore ocean waves, Mar. Technol. Soc. J., 10, 19-25. 